\title{
Application of neural networks for achieving 802.11 QoS in heterogeneous channels
}

\author{
Chiapin Wang ${ }^{\mathrm{a}}$, Tsungnan Lin ${ }^{\mathrm{a}, \mathrm{b}, *}$ \\ a Graduate Institute of Communication Engineering, National Taiwan University, Taipei, Taiwan, ROC \\ b Department of Electrical Engineering, National Taiwan University, No. 1, Section 4, Roosevelt Road, Taipei 106, Taiwan, ROC
}

Received 2 April 2007; received in revised form 22 October 2007; accepted 24 October 2007

Available online 4 November 2007

Responsible Editor: I.F. Akyilidz

\begin{abstract}
In error-prone IEEE 802.11 WLAN (Wireless Local Area Network) environments, heterogeneous link qualities can significantly affect channel utilizations of mobile stations and consequently the user-perceived QoS (Quality of Services) of multimedia applications. In this paper we propose a novel optimization framework which provides QoS by adjusting IWSs (Initial Window Size) according to current channel states and QoS requirements. It is a table-driven approach which offline pre-establishes the table of the best IWSs based on a cost-reward function. Neural networks are utilized to learn the mapping correlation and then to generalize that to other situations of interest. At runtime, the IWS of each user can thus be determined optimally with a simple table lookup rapidly without much time spent on learning about the nonlinear and complicated correlation. A video streaming transmission scenario is used to evaluate the performance of our scheme. The simulation results demonstrate that the proposed mechanism can effectively provide QoS for each user when the capacity of the network is sufficient for the requirements of all users.
\end{abstract}

(C) 2007 Elsevier B.V. All rights reserved.

Keywords: IEEE 802.11 WLANs; QoS; Neural networks

\section{Introduction}

With the popularity of the IEEE 802.11 based WLANs (Wireless Local Area Networks) recently, the demands of multimedia services for mobile users are increasing. Multimedia applications have specific

\footnotetext{
${ }^{*}$ Corresponding author. Address: Department of Electrical Engineering, National Taiwan University, No. 1, Section 4, Roosevelt Road, Taipei 106, Taiwan, ROC.

E-mail address: tsungnan@ntu.edu.tw (T. Lin).
}

requirements in terms of throughput fluctuation, delay sensitivity, loss tolerance, etc. due to their differentiated traffic types. Thus the provisioning of QoS (Quality of Service) in 802.11 WLANs becomes progressively important. The IEEE 802.11 standards [1] of MAC (Medium Access Control) support two fundamental mechanisms for channel arbitration, namely, DCF (Distributed Coordination Function) and PCF (Point Coordination Function). DCF is a random access mechanism based on CSMA/ CA (Carrier Sense Multiple Access/Collision 
Avoidance) protocols, whereas PCF is a centralized scheduling protocol which can provide a contentionfree channel access for time-bounded traffic. Due to the complexity of deploying PCF based WLANs, the current implementations of 802.11 WLANs mostly employ DCF mechanisms. To support QoS in ordinary $802.11 \mathrm{DCF}$, the IEEE 802.11 standardization committee just finished the work on IEEE 802.11e [2]. In the contention-based part, IEEE 802.11e employs a priority scheme, called EDCF (Enhanced Distributed Coordination Function), which differentiates the IFS (inter-frame space) and backoff parameters according to service classes to support real-time applications.

Many previous efforts [3-12] try to improve the performance of 802.11 QoS by finding out the optimal values of some differentiated parameters based on theoretical analyses or simulations. Banchs and Perez [4] extended 802.11 DCF protocols to provide throughput guarantees by adapting the contention window according to the service class. Xiao [6] proposed a backoff-based priority scheme using an analytical model of 802.11e EDCF. Tinnirello et al. [9] investigated on the performance impacts of differentiating initial window size, window increasing factor and IFS, respectively, and then proposed a joined differentiation scheme involving initial window size and IFS. However, most of these works provide solutions with the assumption of ideal-channel conditions or homogeneous link qualities among the participating hosts which is impractical in realistic wireless environments. The transmission qualities of hosts, e.g. their BER (Bit Error Rate) levels, actually are diverse at most of the times even with a link adaptation mechanism applied on 802.11 PHY (physical layer) [1] due to limited MCSs (Modulation and Coding Schemes) available. To consider the performance impact of heterogeneous link qualities on 802.11 QoS, we conduct a simulation scenario of error-prone channels as shown in Section 2. The numerical results show that unequal link qualities among hosts can pose severe unfairness of channel sharing. For example, it is shown that an average variation of throughput due to the difference of transmission qualities between an error-free condition and a BER of $2 \mathrm{E}-5$ can be as large as $48 \%$. This implies that the multimedia QoS of users in adverse channel conditions may be significantly degraded even when the service differentiation mechanism is applied.

Observing that 802.11 QoS depends on not only the applied differentiation mechanism but also the link quality, we are thus motivated to propose a dynamic IWS (initial window size) scheme which compensates skewed channel shares due to heterogeneous channel conditions. A cost-reward function is explored to quantify the performance of QoS. Based on this function, the table of optimal IWSs with respect to channel conditions and QoS requirements can be established in the off-line stage. Neural networks are utilized to learn the mapping correlation and to generalize that to other cases of interest. At runtime, the QoS of each user can thus be achieved by adjusting its IWS optimally with a simple table lookup rapidly without much time spent on learning about the nonlinear and complicated correlation.

We conduct simulations to demonstrate the effectiveness of the proposed scheme based on a video streaming transmission scenario. We examine two representative scenarios of dynamics in WLANs that users experience heterogeneous channel conditions and that users are with different QoS requirements. The simulation results show that the proposed mechanism can effectively tackle varying heterogeneous channel conditions to provide QoS for each user when the capacity of the network is sufficient to satisfy the requirements of all users. The remainder of this paper is organized as follows. Section 2 presents simulation results to exhibit the variation of QoS due to heterogeneous channel conditions. In Section 3, we formalize this problem as an optimization problem and provide our solution of a neural-network-based dynamic IWS scheme. In Section 4, we construct simulation scenarios to evaluate the effectiveness of the proposed scheme. Section 5 draws our conclusions.

\section{Numerical results and discussion}

In this Section, we conduct IEEE 802.11 transmission scenarios of all the users with the same service class while in different channel conditions. Through such the scenarios we can clearly explore the variation of QoS among users due to their heterogeneous channel conditions. The 802.11 simulation model is developed with Matlab codes based on IEEE $802.11 \mathrm{~b}$ standard [1]. This model can be used to evaluate saturated throughput and delay (while a host always has a packet to send) with various values of IWSs in error-prone 802.11 WLANs. In our simulations, we assume a fixed number of 802.11 b contending hosts within the transmission range of each other. Each host transmits a real-time 
traffic flow of an identical class to the corresponding receiver with the basic CSMA/CA scheme. In an illustrated scenario, we assume that half of the hosts, named ideal-channel (IC) hosts, are always in a stationary and ideal-channel condition (i.e. BER $=0$ ). The others, named error-prone-channel (EC) hosts, are initially in an ideal condition and later suffer from channel degradation due to mobility with an average BER of $1 \mathrm{E}-5$ and $2 \mathrm{E}-5$ in sequence. For demonstration purposes, the data rate is $1 \mathrm{Mbps}$ and the transmitting packet length is fixed as 1023 bytes. The system parameters adopted are shown in Table 1.

The evaluation variables are the number of hosts, $K$ and the BER of EC hosts, $B E R(E C)$, while the aggregated throughput and average delay corresponding to IC and EC hosts are presented in Figs. 1 and 2, respectively. It is shown that when all the hosts are in an ideal condition initially, i.e. $B E R(E C)$ is equal to 0 , their performances are equal in terms of both throughput and delay. When $B E R(E C)$ later deteriorates to $1 \mathrm{E}-5$ and $2 \mathrm{E}-5$ consecutively, the performance variation between IC and EC hosts is gradually enlarged. This can therefore pose the degradation of QoS perceived on EC hosts. For example, consider 10 contending hosts with each one demanding a throughput level of $64 \mathrm{Kbps}$ and a delay bound of $120 \mathrm{~ms}$ for satisfying QoS. In case that all the hosts are with ideal channels, their individual throughput is $78.96 \mathrm{Kbps}$ and delay is $103.02 \mathrm{~ms}$ as shown in Figs. 1 and 2, respectively. Thus the user-perceived QoS is assured. If the channel conditions of half hosts deteriorate with BER of $2 \mathrm{E}-5$, the corresponding throughput and delay become $55.86 \mathrm{Kbps}$ and $141.44 \mathrm{~ms}$, respectively, which can no more meet the prescribed QoS. In the meanwhile, the performances of the other hosts keeping in ideal conditions are improved to $94.46 \mathrm{Kbps}$ and $87.23 \mathrm{~ms}$. The average variation of throughput and delay between hosts is as large as $47.6 \%(37.6 \mathrm{Kbps} / 78.96 \mathrm{Kbps}=$ $47.6 \%)$ and $52.6 \%(54.21 \mathrm{~ms} / 103.02 \mathrm{~ms}=52.6 \%)$, respectively. Through these results it is demonstrated that heterogeneous link qualities among users have a significant impact on the perceived QoS under adverse conditions.

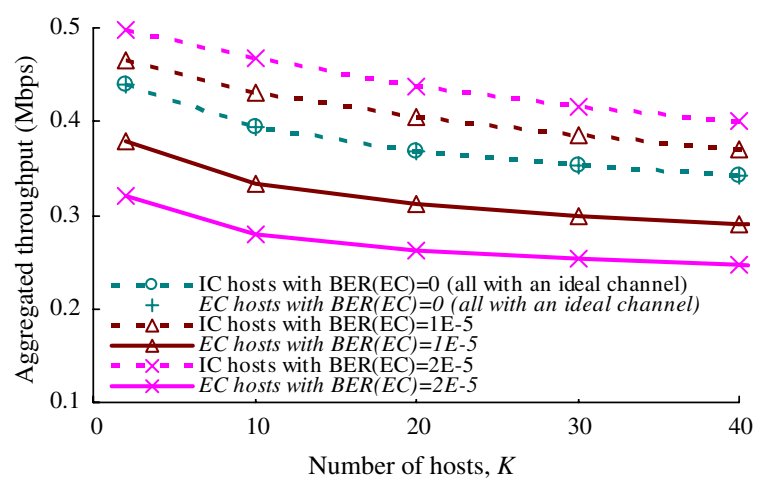

Fig. 1. The aggregated throughput of IC and EC hosts, respectively, vs. the total number of hosts, $K$ varying with the BER level of EC hosts, $B E R(E C)$. For instance, in case of $B E R(E C)$ equal to $1 \mathrm{E}-5$, the aggregated throughput of IC hosts is indexed as the triangle-dotted line, while that of EC hosts is indexed as the triangle-solid line.

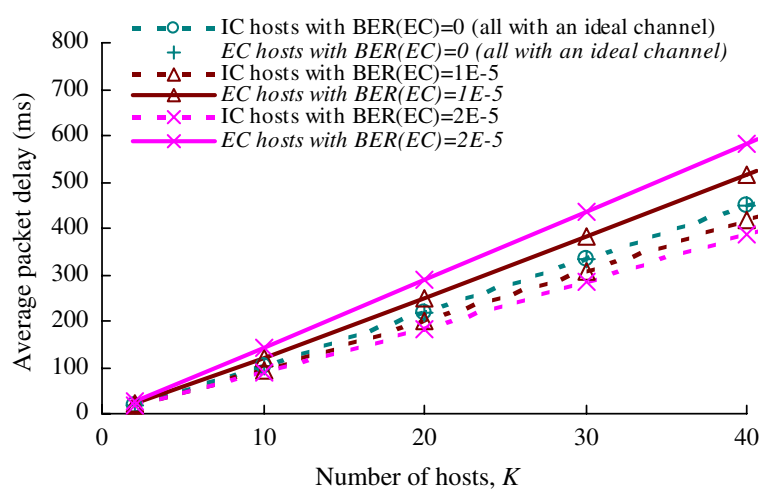

Fig. 2. The average packet delay of IC and EC hosts, respectively, vs. the total number of hosts varying with the BER level of EC hosts.

The performance variation arises as a result of the following facts. Due to its higher BER, an EC host will averagely experience more retries to succeed a transmission than an IC host does. When a retransmission is performed, according to the 802.11 CSMA/CA standards, the backoff window size will be increased exponentially until the retries come to a certain limit. Thus an EC host would averagely adopt a larger backoff timer and then has less chance to access the channel. Such the unfair behavior is

Table 1

System parameters

\begin{tabular}{|c|c|c|c|c|c|}
\hline MAC header & 28 bytes & DIFS (DCF inter-frame space) & $50 \mu \mathrm{s}$ & Initial window size & 32 \\
\hline PHY header & 24 bytes & SIFS (short inter-frame space) & $10 \mu \mathrm{s}$ & Maximum window size & 1024 \\
\hline ACK (acknowledgement) frame & 38 bytes & Slot time & $20 \mu \mathrm{s}$ & Retry limit & 5 \\
\hline
\end{tabular}


similar to the scenarios of asymmetric information among nodes [13]. Thus applying 802.11 CSMA/ CA protocols with fixed system parameters can not assure multimedia QoS in heterogeneous WLAN environments. Alternatively, under adverse conditions, each host needs to optimally adjust its parameters to variable channels conditions.

\section{The proposed neural-network-based optimization framework}

From the observation above, it is shown that QoS cannot be achieved by the approach of fixed parameters in a varying heterogeneous wireless environment. In this Section, we formalize this problem as an optimization problem and present our solution of an optimization framework based on neural networks.

\subsection{Problem formalization}

Consider $K$ users with each one transmitting a real-time traffic flow in IEEE 802.11 WLANs. Assume that the QoS requirement of user $i$ is $Q o S_{-}$$r e q_{i}$ and that the network resource is sufficient to support the requirements of the $K$ users. QoS reqi here can be one of throughput, delay, jitter, packet loss rate, or other QoS metrics of concern depending on the characteristics of the provided applications. We investigate the problem of achieving 802.11 QoS by adjusting the MAC-layer argument, initial window size (IWS). The reason of using IWS is that it is a key parameter affecting not only the access priority but also the overall system performance [9]. The optimal IWSs of the $K$ users can be chosen by minimizing a cost-reward function such that the perceived QoS of the $K$ users are close to their requirements, $Q o S \_r e q_{i}$. The cost-reward function, $C_{\mathrm{Qos}}$ can be expressed as:

$C_{\mathrm{QoS}}=\sum_{i=1}^{K}\left(Q_{o} S_{\text {per }}-\mathrm{QoS}_{-} r e q_{i}\right)^{2}$,

where $Q o S \_p e r_{i}$ is the perceived QoS of user $i$.

The best IWSs which conduct to the minimum value of $C_{\mathrm{QoS}}$ essentially depend on the QoS requirements of users and also the characteristics of communication environments such as the number of users, transmitting data rates, and experienced channel conditions. In this paper we consider a given number of users with fixed data rates, and focus on the problem of achieving QoS in heterogeneous channels. In this sense, we use a following transmission scenario to evaluate the best IWSs associated with different QoS requirements and channel conditions. Assume that two IEEE $802.11 \mathrm{~b}$ users transmit real-time traffic flows with a fixed packet size of 1500 bytes using the data rate of $1 \mathrm{Mbps}$. One user, named ideal-channel (IC) user, is with a given QoS requirement in an ideal-channel condition (i.e. $\mathrm{BER}=0$ ), while the other one, named error-prone-channel (EC) user, is with varied QoS requirements and experiences dynamic channel qualities. We apply a default value of IWS to IC user ( 32 adopted from the IEEE $802.11 \mathrm{~b}$ standards [1]), and adjust the value of IWS from 4 to 64 for EC user. Accordingly we can calculate the value of $C_{\mathrm{QoS}}$ in Eq. (1) with respect to different value of IWS and then derive the best one which provides a minimum value of $C_{\mathrm{Qos}}$.

Fig. 3 presents the numerical results of the costreward function vs. IWS of EC user varying with its BER, $B E R(E C)$ while the throughput requirement for satisfying QoS is $300 \mathrm{Kbps}$ to IC user and $400 \mathrm{Kbps}$ to EC user, respectively. We can see from the results that these curves are concave with a minimum value of cost-reward function and the corresponding IWS. When EC user experiences worse and worse channel conditions, i.e. $B E R(E C)$ gets larger and larger, the best IWS which conducts to a minimum cost-reward function becomes smaller. The reason is that 802.11 users in adverse channel conditions require better system parameters (e.g. small IWS) so as to compensate their channel conditions. When $B E R(E C)$ is $0,2 \mathrm{E}-5,4 \mathrm{E}-5,6 \mathrm{E}-5$ and $8 \mathrm{E}-5$, the best IWS is $26,14,8,5$, and 4 , respectively. Based on the cost-reward function we can evaluate the best IWSs corresponding to diverse channel conditions and also varied situations of

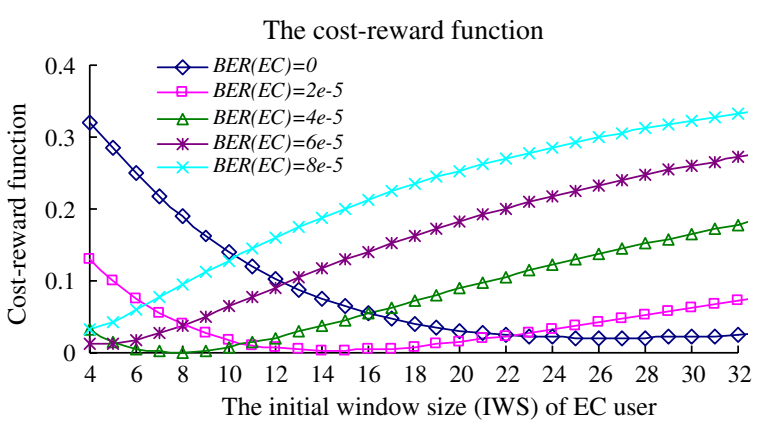

Fig. 3. The cost-reward function vs. IWS of EC user varying with its BER, $B E R(E C)$, in case that the throughput requirement of IC and EC user is $300 \mathrm{Kbps}$ and $400 \mathrm{Kbps}$, respectively. 
Table 2

The optimal IWSs of EC user with respect to its channel conditions and throughput requirements (in case that the throughput requirement of IC user is $300 \mathrm{Kbps}$ ), with 20 pairs of data for training (presented as a regular type) and 5 pairs of data for testing (presented as an italic type)

\begin{tabular}{llllll}
\hline $\begin{array}{l}\text { Throughput } \\
\text { requirements (Kbps) }\end{array}$ & \multicolumn{5}{l}{ Channel conditions (BER) } \\
\cline { 2 - 6 } & 0 & $2 \mathrm{E}-05$ & $4 \mathrm{E}-05$ & $6 \mathrm{E}-05$ & $8 \mathrm{E}-05$ \\
\hline 100 & 47 & 25 & 12 & 7 & 5 \\
200 & 38 & 21 & 11 & 6 & 4 \\
300 & 32 & 17 & 9 & 5 & 4 \\
400 & 26 & 14 & 8 & 5 & 4 \\
500 & 22 & 12 & 6 & 4 & 4 \\
\hline
\end{tabular}

QoS requirements. Table 2 shows the numerical results of EC user's optimal IWSs with respect to its channel conditions and throughput requirements while IC user demands a fixed throughput of $300 \mathrm{Kbps}$ for QoS. Table 3 presents the similar results associated with delay while the delay constraint of IC user is fixed as $30 \mathrm{~ms}$. It can be observed from both Tables 2 and 3 that when the channel condition is worse and worse, or the QoS requirement gets higher and higher, the optimal IWS will become smaller and smaller consequently. The reason is that 802.11 users which are either in adverse channel conditions or with high QoS demands will require superior system parameters (e.g. small IWS) so as to compensate the channel conditions or meet their requirements accordingly. From these results shown in Tables 2 and 3 we can determine the optimal IWS for each user corresponding to its channel conditions and QoS requirements. For example, when an 802.11 user experiences the BER of $2 \mathrm{E}-5$ and is with the throughput demand of $400 \mathrm{Kbps}$ or the delay bound of $30 \mathrm{~ms}$ to meet QoS requirements, the best IWS for it will be 14 and 18, respectively. The question now becomes how to determine the optimal

\section{Table 3}

The optimal IWSs of EC user with respect to its channel conditions and delay constraints (in case that the delay constraint of IC user is $30 \mathrm{~ms}$ ), with 20 pairs of data for training (presented as a regular type) and 5 pairs of data for testing (presented as an italic type)

\begin{tabular}{llllll}
\hline Delay constraint (ms) & \multicolumn{6}{c}{ Channel conditions (BER) } \\
\cline { 2 - 6 } & 0 & $2 \mathrm{E}-05$ & $4 \mathrm{E}-05$ & $6 \mathrm{E}-05$ & $8 \mathrm{E}-05$ \\
\hline 50 & 64 & 38 & 19 & 11 & 8 \\
40 & 54 & 26 & 13 & 8 & 6 \\
30 & 32 & 18 & 10 & 7 & 5 \\
20 & 25 & 16 & 9 & 6 & 4 \\
10 & 22 & 14 & 8 & 6 & 4 \\
\hline
\end{tabular}

IWSs with respect to other unknown channel conditions and QoS requirements.

\subsection{The proposed neural-network-based optimization framework}

Here we propose an optimization framework which adjusts IWS optimally by using an artificial neural network (ANN) to learn the nonlinear I-O correlations among channel conditions, QoS requirements and IWSs, and then to generalize that to other cases of channel conditions and QoS requirements. ANNs have been reported to be effective in modeling the complex relationship between the input signal and the output signal, and have outstanding generalization performance [14]. We adopt the multilayer perceptron (MLP) [15], one of the most popular ANN, to model the correlation function between the optimal IWS and the corresponding channel conditions and QoS requirements. A MLP is composed of an input layer, one or more hidden layers, and an output layer. It learns the nonlinear function by means of a "supervised" manner, i.e. pairs of input and output values are provided in advance and imported into the network for learning the relationship between the input and output. The input signals are taken in the first layer and "fed forward" through the network layer by layer to the output. Thus a MLP can learn the correlation function adaptively by iteratively adjusting its weights and biases in a "back-propagated" direction so as to minimize the errors between the actual outputs and the desired outputs. Such the nonlinear, feed-forward, and back-propagated MLP consists of massively parallel processor with the potential to be fault tolerance [15].

The dashed rectangle of Fig. 4 shows the architecture of the exploited MLP for our framework. It consists of one hidden layer and 2-6-1 sensory units from the input layer to the output. The input signals, i.e. the channel condition and QoS requirement (throughput requirement or delay constraint) are taken in the input layer and propagate through the network layer by layer to the output. The $i$ th neuron at the $l$ th layer can be described as:

$u_{i}(l)=\sum_{j=1}^{N_{l-1}} \omega_{i j}(l) a_{j}(l-1)+\theta_{i}(l)$,
$a_{i}(l)=h_{l}\left(u_{i}(l)\right) 1 \leqslant i \leqslant N_{l} ; \quad l=1,2$,

where $N_{l}$ is the number of neurons at the $l$ th layer; $u_{i}(l)$ and $a_{i}(l)$ are correspondingly the activation 


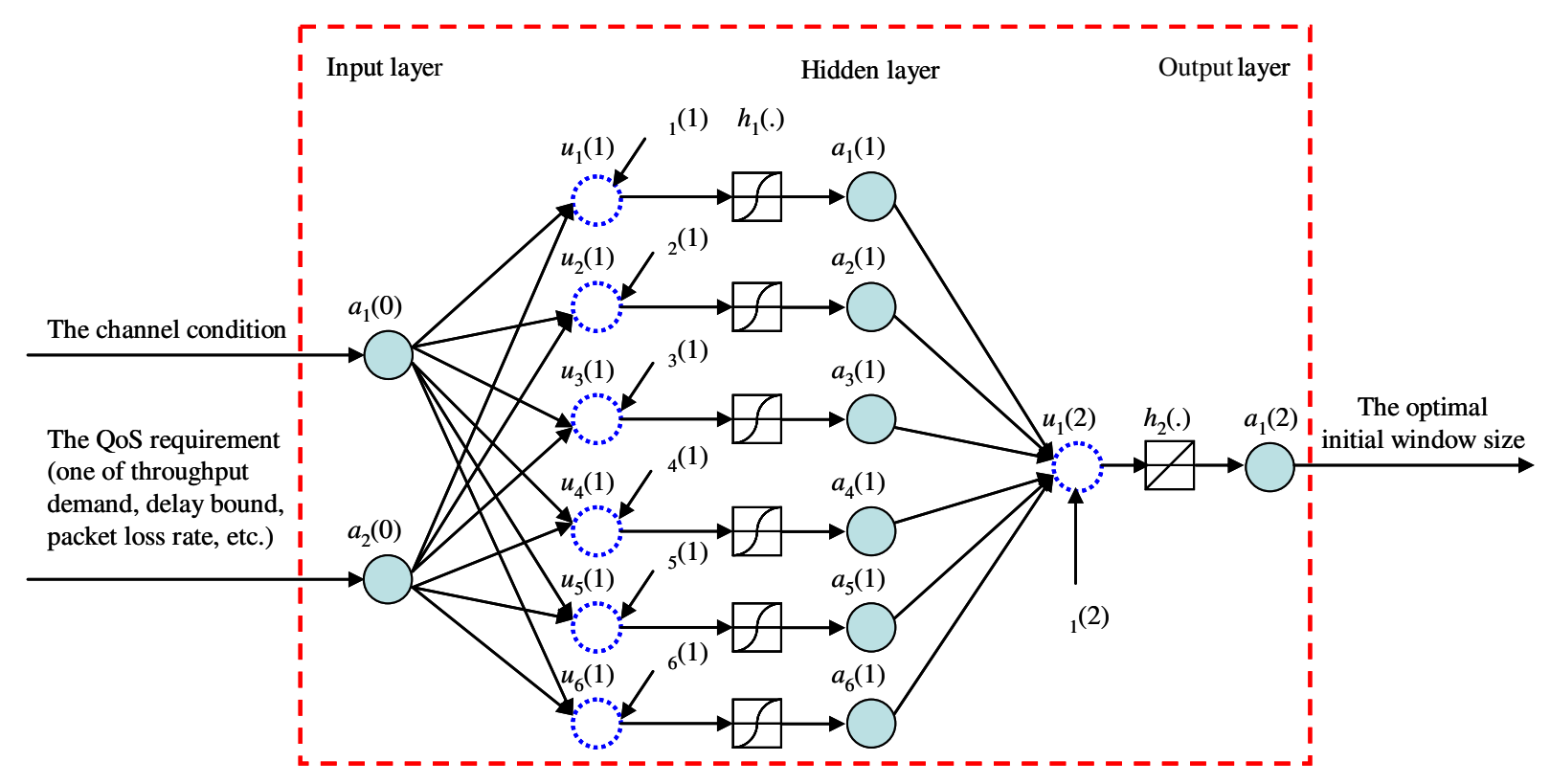

Fig. 4. The exploited neural network architecture.

and output values of the $i$ th neuron at the $l$ th layer. The input units are represented by $a_{i}(0)$ and the output units by $a_{i}$ Eq. (2). $\omega_{i j}(l)$ refers to the weight connecting the output of the $j$ th neuron at the $(l-1)$ th layer to the activation of the $i$ th neuron at the lth layer. $\theta_{i}(l)$ refers to the bias associated with the $i$ th neuron at the $l$ th layer. $h_{l}(\cdot)$ is the transfer function between the activation and output of the neuron.

The nonlinear function can be modeled with MLP by recursively adjusting $\omega_{i j}(l)$ and $\theta_{i}(l)$ to minimize the mean squares error (MSE) between the targets, $\operatorname{tar}_{i}$ (the optimal IWS in this case) and actual outputs, $a_{i}$ Eq. (2). That is,

$E=\frac{1}{2} \sum_{m=1}^{M} \sum_{i=1}^{N_{2}}\left(\operatorname{tar}_{i}^{(m)}-a_{i}^{(m)}(2)\right)^{2}$,

where $M$ is the number of teacher patterns. The universal approximation theorem [14] shows that MLP can approximate the nonlinear function to an arbitrary degree of the accuracy. The learning and generalizing processes of MLP in detail can be seen in [15].

We implement the exploited MLP shown in Fig. 4 by Matlab 6.0 Neural Network Toolbox [16]. The selected training function is the Levenberg-Marquardt (LM) algorithm $[17,18]$ which is a standard technique for nonlinear least squares problems. The used gradient-descent-momentum
(GDM) learning function [19] updates weight and bias values according to gradient descents with momentums. The transfer function $h_{l}(\cdot)$ in hidden layer $(l=1)$ and output layer $(l=2)$ is a hyperbolic tangent sigmoid function, $h_{l}(x)=2 /(1+\exp (-2 x))$ -1 , and a linear transfer function, $h_{l}(x)=x$, respectively. We set the epoch (i.e. the number of times for adjusting the weights and biases) as 100 with the learning rate (the step size of adjusting the weights and biases) of 0.02 .

The teacher patterns associated with the QoS requirements in terms of throughput demands and delay constraints, respectively, are provided in Tables 2 and 3. In each table, we have 25 pairs of input vectors (indexed as channel conditions, QoS requirements) and corresponding output IWSs, with 20 pairs of data for training and 5 pairs of data for testing. The training data of Tables 2 and 3 are imported into MLP to learn the nonlinear function, respectively, while their MSEs vs. training epochs are depicted in Figs. 5 and 6. It is shown that in the end of training, the MSE with Tables 2 and 3 comes to $1.612 \mathrm{E}-4$ and $3.276 \mathrm{E}-5$, respectively. By testing, the MSE is $4.756 \mathrm{E}-4$ and $9.847 \mathrm{E}-6$, respectively. Both of the training and testing errors are low sufficiently to provide an accurate learning result.

With the trained and tested MLP, the I-O correlations can be generalized for other input signals (i.e. channel conditions and QoS requirements) 


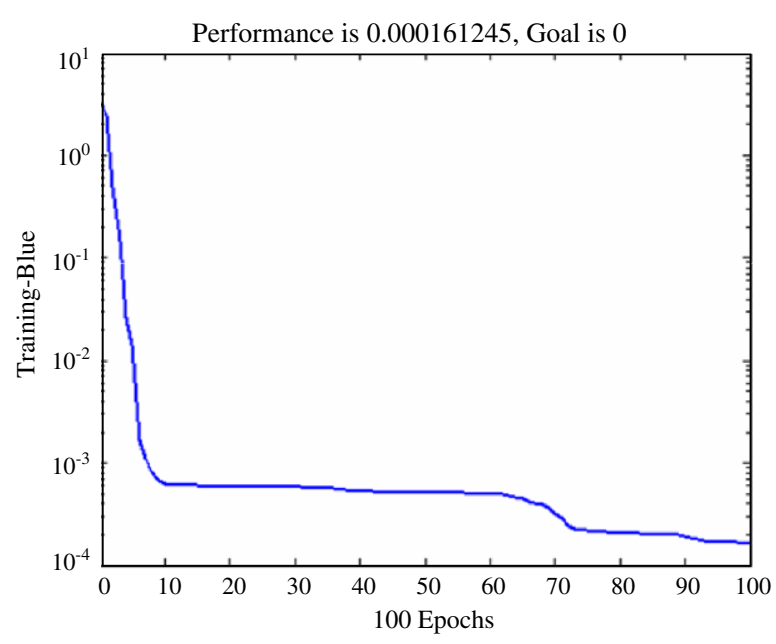

Fig. 5. The plot of MSE vs. epoch associated with the training data in Table 2.

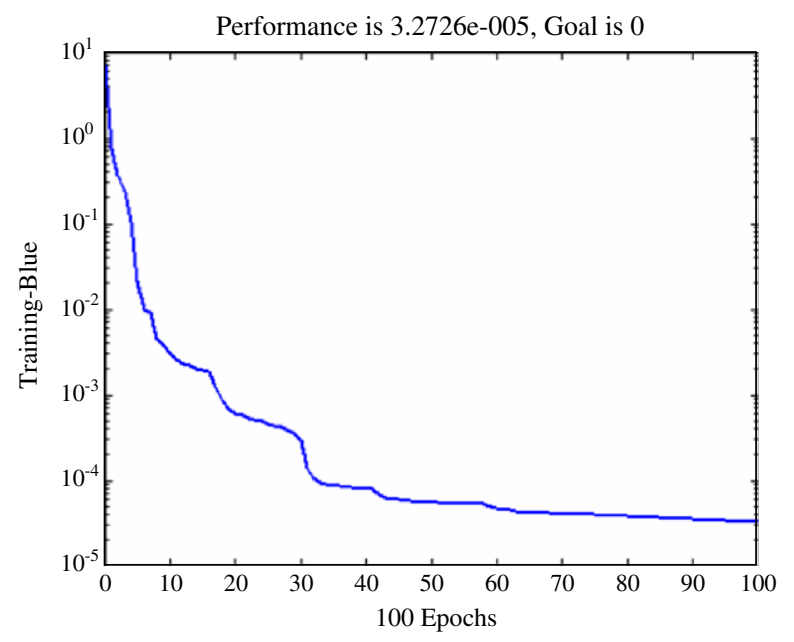

Fig. 6. The plot of MSE vs. epoch associated with the training data in Table 3. and some results are shown in Tables 4 and 5. By comparing Tables 2 and 4 as well as Tables 3 and 5 it can be observed that the MLP outputs and the desired outputs are very close, and will be the same while the values of MLP outputs are approximated with the closest integer. In this sense, the I$\mathrm{O}$ correlations can be generalized to other cases of interest and thus IWS of each user can be adjusted optimally according to its situations with a lookup mapping table.

The proposed ANN-based table-driven scheme adjusts each 802.11 station's IWS to provide QoS fairness in a distributed sense and thus will be implemented in the station site. When the off-line learning process is accomplished, the generalized I-O mapping tables will be built in the stations. At runtime, the transmitting station can estimate the channel conditions based on the measurements about the current SNR (Signal to Noise Ratio), or by way of calculating the number of unacknowledged MAC frame transmissions over a sample time periodically [20]. Practically, the sample time of measurements should be less than the channel coherence time, i.e. the time period within which the wireless channel characteristic can be regarded unchanged, in order to perform an accurate and timely channel probe. At a mobile speed of $1 \mathrm{~m} / \mathrm{s}$ (nearly the walking speed) in IEEE $802.11 \mathrm{~b}$ WLANs with the center frequency of $2.4 \mathrm{GHz}$, the channel coherence time is about $120 \mathrm{~ms}$ [21]. Thus the coherence time of 802.11 wireless channels is generally on the order of multiple packet transmission times in practice [21]. This indicates that these measurement-based approaches have the potential to perform an accurate and timely probing of 802.11 channels. With the updated information of

Table 4

The generalized I-O mapping of (channel conditions, throughput requirements) and optimal IWS

\begin{tabular}{|c|c|c|c|c|c|c|c|c|c|c|c|c|c|c|c|c|c|}
\hline \multirow{2}{*}{$\begin{array}{l}\text { Throughput } \\
\text { requirements } \\
(\mathrm{Kbps})\end{array}$} & \multicolumn{17}{|c|}{ Channel conditions $(\mathrm{BER})(\times 1 \mathrm{E}-5)$} \\
\hline & 0 & 0.5 & 1 & 1.5 & 2 & 2.5 & 3 & 3.5 & 4 & 4.5 & 5 & 5.5 & 6 & 6.5 & 7 & 7.5 & 8 \\
\hline 100 & 47.0 & 40.3 & 34.2 & 29.2 & 25.1 & 21.4 & 16.2 & 13.8 & 11.9 & 10.4 & 9.2 & 8.2 & 7.0 & 5.9 & 5.7 & 5.3 & 5.0 \\
\hline 150 & 41.7 & 35.7 & 30.9 & 26.9 & 23.5 & 20.0 & 15.5 & 13.3 & 11.3 & 9.6 & 8.4 & 7.3 & 5.6 & 4.4 & 4.3 & 4.7 & 4.7 \\
\hline 200 & 38.0 & 31.4 & 27.2 & 23.8 & 21.0 & 17.5 & 13.8 & 12.2 & 10.9 & 9.9 & 9.0 & 7.7 & 6.0 & 5.1 & 4.3 & 3.7 & 4.0 \\
\hline 250 & 35.2 & 28.3 & 24.1 & 21.2 & 18.8 & 15.2 & 12.4 & 11.0 & 10.0 & 9.1 & 8.2 & 6.5 & 5.5 & 5.0 & 4.8 & 4.5 & 4.4 \\
\hline 300 & 32.0 & 26.9 & 21.7 & 19.0 & 17.0 & 13.2 & 11.2 & 10.1 & 9.2 & 8.4 & 7.1 & 5.6 & 5.1 & 4.8 & 4.5 & 4.4 & 4.2 \\
\hline 350 & 28.8 & 25.5 & 20.4 & 17.3 & 15.4 & 11.7 & 10.2 & 9.3 & 8.5 & 7.6 & 6.0 & 5.2 & 4.8 & 4.6 & 4.4 & 4.2 & 4.1 \\
\hline 400 & 26.1 & 23.6 & 20.4 & 16.1 & 14.0 & 10.5 & 9.4 & 8.6 & 7.9 & 6.5 & 5.3 & 4.8 & 4.6 & 4.4 & 4.2 & 4.1 & 4.0 \\
\hline 450 & 23.8 & 21.7 & 19.8 & 16.2 & 12.8 & 9.5 & 8.7 & 8.0 & 7.1 & 5.5 & 4.9 & 4.6 & 4.4 & 4.2 & 4.1 & 4.0 & 3.9 \\
\hline 500 & 21.9 & 20.2 & 18.7 & 16.8 & 12.0 & 8.8 & 8.1 & 7.4 & 6.0 & 5.0 & 4.6 & 4.4 & 4.3 & 4.1 & 4.0 & 3.9 & 3.9 \\
\hline 550 & 20.3 & 18.8 & 17.7 & 16.6 & 12.4 & 8.4 & 7.6 & 6.6 & 5.2 & 4.7 & 4.4 & 4.3 & 4.1 & 4.0 & 3.9 & 3.9 & 3.8 \\
\hline
\end{tabular}


Table 5

The generalized I-O mapping of (channel conditions, delay constraints) and optimal IWS

\begin{tabular}{|c|c|c|c|c|c|c|c|c|c|c|c|c|c|c|c|c|c|}
\hline \multirow[t]{2}{*}{ Delay constraint (ms) } & \multicolumn{17}{|c|}{ Channel conditions $(\mathrm{BER})(\times 1 \mathrm{E}-5)$} \\
\hline & 0 & 0.5 & 1 & 1.5 & 2 & 2.5 & 3 & 3.5 & 4 & 4.5 & 5 & 5.5 & 6 & 6.5 & 7 & 7.5 & 8 \\
\hline 50 & 64.0 & 59.5 & 53.5 & 45.7 & 38.0 & 31.7 & 26.6 & 22.5 & 19.1 & 16.3 & 14.0 & 12.2 & 10.9 & 9.8 & 9.1 & 8.5 & 8.1 \\
\hline 45 & 56.1 & 49.9 & 42.7 & 34.6 & 26.9 & 21.3 & 17.5 & 14.8 & 12.7 & 11.2 & 10.1 & 9.2 & 8.5 & 8.1 & 7.7 & 7.4 & 7.2 \\
\hline 40 & 54.0 & 47.6 & 40.8 & 33.2 & 26.0 & 20.8 & 17.3 & 14.8 & 12.9 & 11.3 & 10.1 & 9.0 & 8.1 & 7.4 & 6.8 & 6.3 & 5.9 \\
\hline 35 & 45.3 & 41.8 & 37.5 & 32.0 & 26.4 & 22.3 & 19.6 & 17.7 & 16.0 & 14.5 & 13.1 & 11.7 & 10.4 & 9.1 & 7.8 & 6.5 & 5.3 \\
\hline 30 & 32.0 & 30.0 & 27.1 & 22.8 & 18.0 & 14.5 & 12.4 & 11.0 & 10.0 & 9.1 & 8.3 & 7.7 & 7.1 & 6.5 & 6.0 & 5.5 & 5.0 \\
\hline 25 & 27.6 & 26.5 & 24.4 & 20.8 & 16.4 & 13.0 & 11.0 & 9.6 & 8.6 & 7.7 & 6.9 & 6.2 & 5.6 & 5.1 & 4.6 & 4.2 & 3.9 \\
\hline 20 & 25.0 & 24.4 & 23.0 & 19.9 & 16.0 & 12.9 & 11.1 & 9.9 & 9.0 & 8.2 & 7.4 & 6.7 & 6.0 & 5.4 & 4.8 & 4.4 & 4.0 \\
\hline 15 & 23.1 & 22.6 & 21.4 & 18.7 & 14.9 & 12.0 & 10.4 & 9.5 & 8.9 & 8.2 & 7.6 & 7.0 & 6.3 & 5.7 & 5.2 & 4.7 & 4.2 \\
\hline 10 & 22.0 & 21.5 & 20.3 & 17.7 & 14.0 & 11.0 & 9.4 & 8.6 & 8.0 & 7.5 & 7.0 & 6.5 & 6.0 & 5.4 & 4.9 & 4.4 & 4.0 \\
\hline 5 & 21.6 & 21.2 & 20.0 & 17.5 & 13.8 & 10.6 & 8.9 & 8.0 & 7.4 & 6.9 & 6.4 & 5.9 & 5.3 & 4.8 & 4.2 & 3.7 & 3.2 \\
\hline
\end{tabular}

current channel conditions and QoS requirements, the station can rapidly adjust its IWS optimally using the look-up mapping tables without much time spent on learning about the nonlinear and complicated correlation.

\section{The performance evaluation and results}

In this Section we conduct simulations to demonstrate the effectiveness of the proposed scheme based on a video transmission scenario. The used IEEE $802.11 \mathrm{~b}$ simulation model is the same as that described in Section 2. We provide two representative scenarios of dynamics in WLANs that users experience heterogeneous channel conditions and that users are with different QoS requirements. In both scenarios we assume a fixed number of pairs of sender and receiver stations to guarantee sufficient bandwidth for overall QoS requirements. All the sender hosts transmit a variable bit rate video streaming consisted of a uniformly spaced sequence of 1500-byte packets to the corresponding receiver hosts with the data rate of $2 \mathrm{Mbps}$. The packet spacing is $1 / 30$ th per second, to emulate the video frame rate of 30 frames/s [22]. The sender hosts consist of the hosts in ideal channels (IC) and those in errorprone-channels (EC) with a BER level, $B E R(E C)$. The number of pairs of packet transmissions is fixed as 4 while the number of EC sender hosts, $n$, is the evaluation parameter. In each simulation scenario, we provide performance analyses while QoS is indexed as throughput demands or delay bounds, respectively. With the proposed scheme, the IWS of each user is determined according to its QoS requirement and channel condition with Tables 4 and 5 , and the chosen values will be rounded to the closest integer. We compare the proposed scheme with IEEE 802.11 DCF which adopts a fixed IWS of 32 in the standards [1].

\subsection{Users are with identical QoS requirements in diverse channel conditions}

In the first scenario, users are with an identical throughput demand or delay bound to meet their QoS requirements while they are under diverse channel conditions. Through such the transmission scenario we can clearly show the effectiveness of our approach providing QoS for all users by optimizing their initial window sizes. Fig. 7 shows the simulation results of throughputs with respect to IC and EC user while their requirements are the same as $350 \mathrm{Kbps}$ and $B E R(E C)$ is equal to $1 \mathrm{E}-5$; Fig. 8 shows the results of delay when the delay bound for QoS is $35 \mathrm{~ms}$ and $\operatorname{BER}(E C)$ becomes worse as $2 \mathrm{E}-5$. Note that the result of $\mathrm{EC}$ and IC user is not available in cases of $n=0(n$ is the number of EC users; only IC users are present) and $n=4$ (only EC users are present), respectively. It is shown in Figs. 7 and 8 that with DCF, when all sending hosts are in perfect channels $(n=0)$, the throughput is $429 \mathrm{Kbps}$ and the delay is $27.95 \mathrm{~ms}$. Hence QoS for video streaming is assured in receiver hosts. While the BERs of some EC sender hosts deteriorate as $1 \mathrm{E}-5$ ( $n$ is 1 and 2 ), the throughput of these hosts drops below $350 \mathrm{Kbps}$ and can no more meet the requirement for QoS. Similarly, it is shown in Fig. 8 that when EC hosts experience worse BERs of $2 \mathrm{E}-5$, their delay becomes larger than $35 \mathrm{~ms}$ in most cases ( is 1, 2, and 3) and cannot satisfy QoS, neither. The QoS degradation of EC hosts is posed by unfair 
QoS performance in terms of throughput

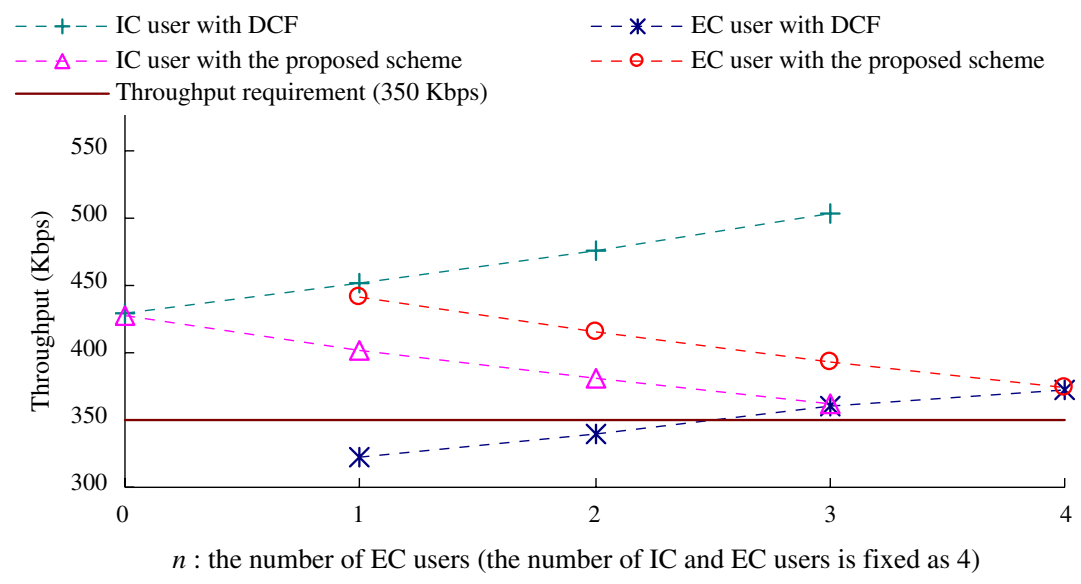

Fig. 7. In the first scenario that the throughput requirement for IC and EC user is the same as $350 \mathrm{Kbps}$, the average throughput of IC user $(\mathrm{BER}=0)$ and $\mathrm{EC}$ user $(\mathrm{BER}=1 \mathrm{E}-5)$ with $802.11 \mathrm{DCF}$ and the proposed scheme, respectively.

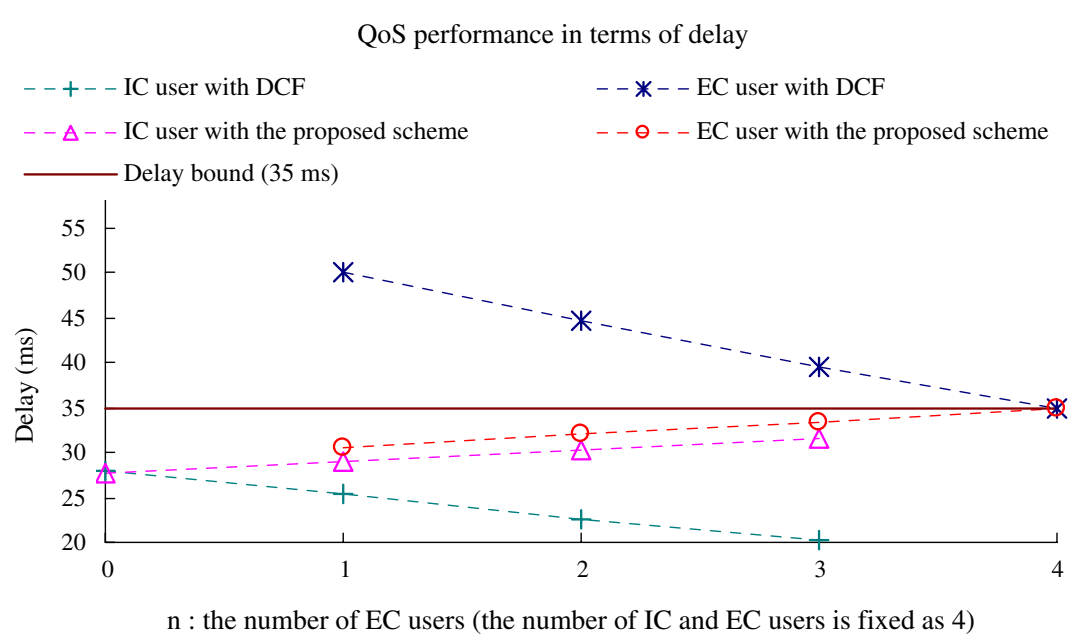

Fig. 8. In the first scenario that the delay bound for IC and EC user is the same as $35 \mathrm{~ms}$, the average delay of IC user (BER $=0$ ) and EC user $(\mathrm{BER}=2 \mathrm{E}-5)$ with $802.11 \mathrm{DCF}$ and the proposed scheme, respectively.

channel sharing due to using fixed parameters under heterogeneous channel conditions.

With the proposed scheme, the value of IWS is adjusted according to the channel conditions and QoS requirements with Tables 4 and 5. Consequently the IWS of EC and IC sender host is differentiated as 20 and 29 , respectively, when the throughput demand is $350 \mathrm{Kbps}$, and differentiated as 26 and 45, respectively, when the delay bound is $35 \mathrm{~ms}$. It is shown that with the proposed scheme, the performances of throughput and delay of EC users are significantly improved. The average throughput of EC hosts is increased approximately by $10.5 \%$ while the delay is reduced by $15.8 \%$ such that the prescribed QoS can be satisfied for each IC and EC user in all cases of $n$. It is shown in Fig. 7 that the throughput performance of EC user is somewhat superior to that of IC user. The reason is that IWSs in practice can only be adjusted with integers and the chosen value will be rounded to the closest integer in Tables 4 and 5 by approximation. Nevertheless, our approach still can effectively minimize the performance variation among users due to diverse channels and the results are presented in Table 6. It is shown that with the proposed scheme, the normalized standard deviation of 
Table 6

In the first scenario, the normalized standard deviation (normalized with the mean) of throughput and delay with $802.11 \mathrm{DCF}$ and the proposed scheme, respectively

\begin{tabular}{lllrrr}
\hline The number of EC users: $n$ & & 0 & 1 & 2 & 3 \\
\hline 802.11 DCF & Normalized standard deviation of throughput (\%) & 0 & 13.4 & 16.6 & 15.6 \\
& Normalized standard deviation of delay (\%) & 0 & 34.1 & 32.7 & 24.2 \\
\multirow{3}{*}{ The proposed scheme } & Normalized standard deviation of throughput (\%) & 0 & 4.0 & 4.4 & 3.6 \\
& Normalized standard deviation of delay (\%) & 0 & 2.3 & 2.8 & 2.5 \\
\hline
\end{tabular}

throughput is reduced from $9.1 \%$ to $2.4 \%$ and that of delay is reduced from $18.2 \%$ to $1.5 \%$ in average. These results demonstrate that the proposed approach can effectively adjust the initial window size to provide fair multimedia QoS for users in varying heterogeneous channels.

\subsection{Users are with different QoS requirements in diverse channel conditions}

In the second scenario, IC and EC users are with different throughput demands or delay constraints to meet their QoS requirements. Fig. 9 presents

QoS performance in terms of throughput

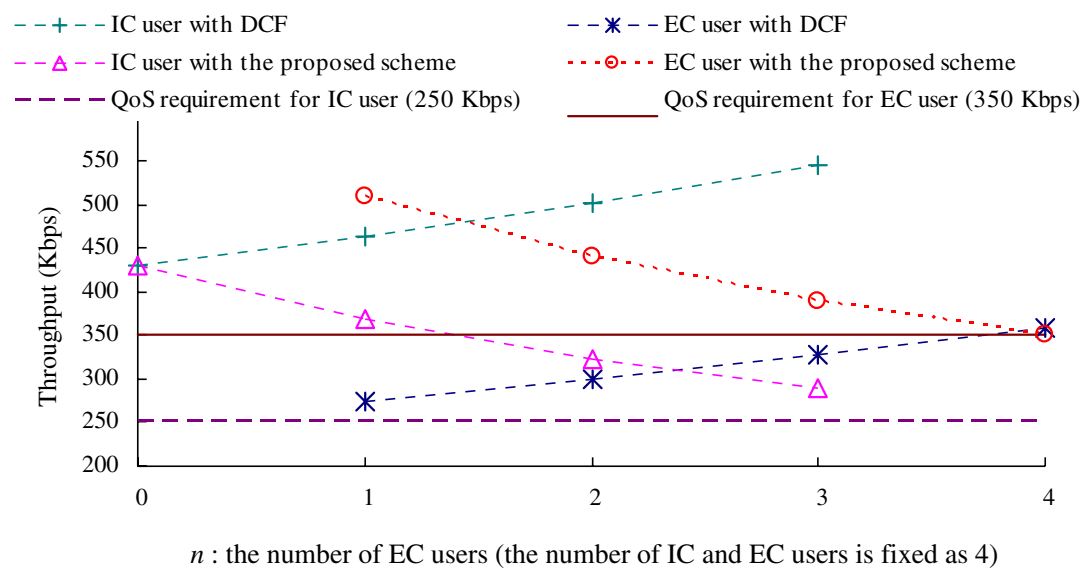

Fig. 9. In the second scenario that the throughput requirement of IC and EC user is $250 \mathrm{Kbps}$ and $350 \mathrm{Kbps}$, respectively, the average throughput of IC user $(\mathrm{BER}=0)$ and $\mathrm{EC}$ user $(\mathrm{BER}=1.5 \mathrm{E}-5)$ with $802.11 \mathrm{DCF}$ and the proposed scheme, respectively.

QoS performance in terms of delay

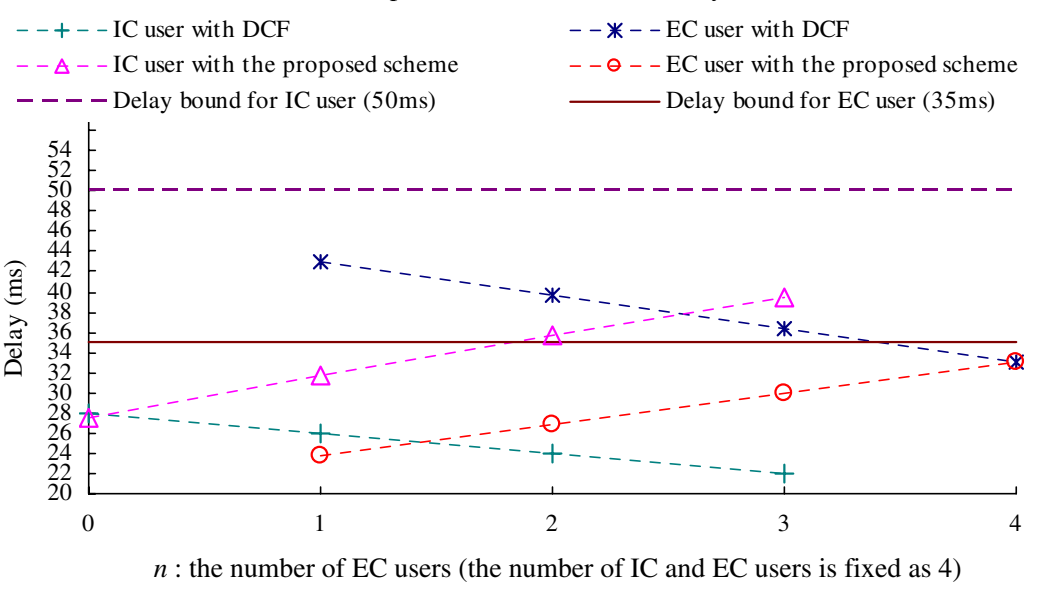

Fig. 10. In the second scenario that the delay bound for IC and EC user is $50 \mathrm{~ms}$ and $35 \mathrm{~ms}$, respectively, the average delay of IC user $(\mathrm{BER}=0)$ and $\mathrm{EC}$ user $(\mathrm{BER}=1.5 \mathrm{E}-5)$ with $802.11 \mathrm{DCF}$ and the proposed scheme, respectively. 
the simulation results of throughputs corresponding to the requirement of IC and EC user being $250 \mathrm{Kbps}$ and $350 \mathrm{Kbps}$, respectively, in case of $B E R(E C)$ equal to $1.5 \mathrm{E}-5$. With $802.11 \mathrm{DCF}$, the average throughput of EC user in most cases ( $n$ is 1,2 , and 3) drops below $350 \mathrm{Kbps}$ and cannot meet the QoS requirement. In the meanwhile, the throughput of IC user much exceeds its demand (250 Kbps). It is shown that a user (EC user) with higher QoS requirements however in adverse channel conditions can suffer from poorer performance than that (IC user) demanding lower QoS in better channels. With the proposed scheme, the IWS of IC and EC user is adjusted as 35 and 17, respectively according to Table 4 . Consequently the throughput performance of EC user is greatly improved such that QoS for each IC and EC user can therefore be satisfied in all cases of $n$. Fig. 10 presents the simulation result of delays corresponding to the bound of IC and EC user being $50 \mathrm{~ms}$ and $35 \mathrm{~ms}$, respectively in case of $B E R(E C)$ equal to $1.5 \mathrm{E}-5$. The result is similar to what is shown in Fig. 9, that with 802.11 DCF, IC user has better channel usage beyond the requirement (its packet delay is further lower than the bound of $50 \mathrm{~ms}$ ) whereas EC user cannot meet the requirement $(35 \mathrm{~ms})$. With the proposed scheme which differentiates the IWS of IC and EC user as 64 and 32, respectively, according to Table 5, both IC and EC user can satisfy their own QoS requirement. It is demonstrated with these simulation results that the proposed approach can effectively tackle the skewed channel sharing problem to provide multimedia QoS in varying heterogeneous WLANs environments.

\section{Conclusion}

We propose in this paper a dynamic IWS (initial window size) scheme for providing multimedia QoS in IEEE 802.11 WLAN environments with heterogeneous channel conditions. In the off-line stage, the table of the best IWSs with respect to channel conditions and QoS requirements is pre-established based on a cost-reward function. Neural networks are utilized to learn the nonlinear mapping function and to generalize that to other cases of interest. At runtime, the QoS of each user can thus be achieved by adjusting its IWS optimally with a simple table lookup rapidly without much time spent on learning about the nonlinear and complicated function. The simulation results of video streaming transmissions demonstrate that the proposed mechanism can effectively provide QoS on each user if the capacity of the network is sufficient for the requirements of all users.

In this paper we focus on a simplified QoS scenario that the requirements of all users are expressed with a given performance metric (such as throughput or delay, respectively), and are only differentiated according to IC and EC users. In the future, we will extend our ANN-based optimization approach for a more general scenario in which users are under various channel conditions with differentiated QoS requirements arbitrarily.

\section{Acknowledgment}

The authors would like to thank the anonymous reviewers for their thoughtful comments and suggestions which have advanced the quality of this paper. This work was supported in part by Taiwan National Science Council under grant 96-2221-E002-091 and 96-2219-E-002-006.

\section{References}

[1] IEEE 802.11a/b, Wireless LAN Medium Access Control (MAC) and Physical Layer (PHY) Specifications, Standard, IEEE, August 1999.

[2] IEEE 802.11 WG, IEEE Standard 802.11e/D8.0, Draft Amendment to STANDARD for Part 11: Wireless Medium Access Control (MAC) and Physical Layer (PHY) specifications: Medium Access Control (MAC) Quality of Service (QoS) Enhancements, February 2004.

[3] D. Deng, R. Chang, A priority scheme for IEEE 802.11 DCF access method, IEICE Transaction on Communications E82B (1) (1999) 96-102.

[4] A. Banchs, X. Perez, Providing throughput guarantees in IEEE 802.11 wireless LAN, in: Proc. of IEEE WCNC, vol. 1, 2002, pp. 130-138.

[5] N.H. Vaidya, P. Bahl, S. Gupta, Distributed fair scheduling in wireless LAN, in: Proc. of ACM MOBICOM 2000, pp. $167-178$

[6] Y. Xiao, A simple and effective priority scheme for IEEE 802.11, IEEE Communications Letters 7 (2) (2003) 70-72.

[7] I. Aad, C. Castelluccia, Differentiation mechanisms for IEEE 802.11, in: Proc. of IEEE INFOCOM 2001, vol. 1, pp. 209 218.

[8] Y. Xue, K. Chen, K. Nahrstedt, Proportional delay differentiation in wireless LAN, in: Proc. of IEEE ICC 2004, vol. 7, pp. 4367-4371.

[9] I. Tinnirello, G. Bianchi, L. Scalia, Performance evaluation of differentiated access mechanisms effectiveness in 802.11 networks, in: Proc. of IEEE GLOBECOM 2004, vol. 5, pp. 3007-3011.

[10] C. Wang, T. Lin, Improving 802.11 QoS in Heterogeneous channels using an adaptive scheme, in: Proc. of IASTED WNET (Wireless Networks and Emerging Technologies), 2006 . 
[11] C. Wang, T. Lin, A neural network based adaptive algorithm for multimedia quality fairness in WLAN environments, in: Proc. of IEEE ICME 2006, pp. 1233-1236.

[12] Y. Yang, R. Kravets, Achieving delay guarantees in Ad Hoc networks through dynamic contention window adaptation, in: Proc. of IEEE INFOCOM 2006, pp. 1-12.

[13] V. Kanodia, A. Sabharwal, B. Sadeghi, E. Knightly, Ordered packet scheduling in wireless ad hoc networks: mechanisms and performance analysis, in: Proc. of ACM MOBIHOC 2002, pp. 58-70.

[14] K. Hornik, M. Stinchcombe, H. White, Multilayer feedforward networks are universal approximators, Neural Networks 2 (1989) 359-366.

[15] S. Haykin, Neural Networks: A Comprehensive Foundation, second ed., Prentice-Hall, 1999.

[16] The Matlab Home Page. http://www.mathworks.com/.

[17] K. Levenberg, A method for the solution of certain nonlinear problems in least squares, Quarterly of Applied Mathematics 2 (2) (1944) 164-168.

[18] D.W. Marquardt, An algorithm for the least-squares estimation of nonlinear parameters, SIAM Journal of Applied Mathematics 11 (2) (1963) 431-441.

[19] N. Qian, On the momentum term in gradient descent learning algorithms, Neural Networks 12 (1999) 145-151.

[20] M.v.d. Schaar, S. Krishnamachari, S. Choi, X. Xu, Adaptive cross-layer protection strategies for robust scalable video transmission over 802.11 WLANs, IEEE Journal on Selected Areas in Communications 21 (10) (2003) 1752-1763.

[21] B. Sadeghi, V. Kanodia, A. Sabharwal, E. Knightly, Opportunistic media access for multirate ad hoc networks, in: Proc. of ACM MOBICOM 2002, pp. 24-35.

[22] Y. Pei and J. Modestino, Multilayered video transmission over wireless channels using an adaptive modulation and coding scheme, in: Proc. of IEEE ICIP 2000, pp. 1009-1012.

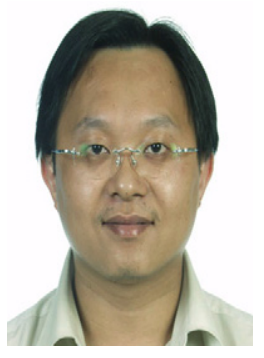

Chiapin Wang was born in Kaohsiung, Taiwan, in 1972. He received the B.S. degree in electrical engineering from National Cheng Kung University, Taiwan in 1994, and the M.S. degree in communication engineering from National Taiwan University, Taiwan in 2003. He is currently working toward the Ph.D. degree at the graduate institute of communication engineering, National Taiwan University, Taiwan. His current research interests include performance analysis and QoS-oriented design for wireless communication networks. He is an IEEE Student Member.

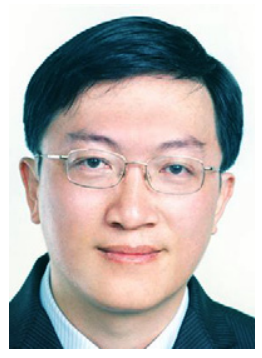

Tsung-Nan Lin received the B.S. degree in electrical engineering from National Taiwan University, Taipei, Taiwan, R.O.C. in 1989, and the M.A. and Ph.D. degrees in electrical engineering from Princeton University, Princeton, NJ, in 1993 and 1996, respectively. He was a Teaching Assistant with the Department of Electrical Engineering, Princeton University, from 1991 to 1992. From 1992 to 1996, he was a Research Assistant with NEC Research Institute. He has been with EPSON R\&D Incorporated and EMC. Since February 2002, he has been with the Department of Electrical Engineering and the Graduate Institute of Communication Engineering, National Taiwan University. He is a Member of Phi Tau Phi scholastic honor society. 